\title{
Das Unplanbare planen
}

\section{Elena Ibello}

palliative $z h+s h$

\author{
Fragen rund um Patientenverfügungen beschäftigten rund 150 Fachpersonen an \\ der Tagung von palliative $\mathrm{zh}+\mathrm{sh}$ in Zürich. Die Teilnehmenden diskutierten und \\ referierten angeregt über Problemstellungen und Lösungsansätze.
}

«Welches Recht und welche Möglichkeiten haben unsere Patienten und Patientinnen, eigene Entscheidungen in Bezug auf das Leben und das Lebensende zu treffen und durchzusetzen?», fragte Monika Obrist zum Einstieg in die Tagung vom 25. Juni 2015. Die CoPräsidentin und Geschäftsleiterin von palliative $\mathrm{zh}+\mathrm{sh}$ eröffnete damit die Diskussionen rund um die Entstehung und Umsetzung von Patientenverfügungen. Auf der Agenda der Tagung standen Fragen wie: Wie nützlich sind Patientenverfügungen? Spiegeln sie die Wünsche und Bedürfnisse der Betroffenen und geben sie klare und realistische Handlungsanweisungen an die Fachleute, wenn es darauf ankommt? Des Weiteren sollten die Rechtsgrundlagen sowie weitere Rahmenbedingungen angesprochen werden, nötige Kompetenzen, die vorhandenen Lücken und damit auch der Handlungsbedarf im Bereich der Patientenverfügungen aufgezeigt werden.

In einem Grusswort richtete Josef Widler, Präsident der Ärztegesellschaft des Kantons Zürich, sein Augenmerk auf das Handeln in der Praxis. Die Patientenverfügung, so Widler, bleibe letztendlich nicht mehr als ein Papier. Was es aber brauche, wenn man Patientinnen und Patienten an ihrem Lebensende betreue, sei Vertrauen. Und meinte damit in erster Linie das Vertrauen von Betroffenen in die Fachpersonen. Widler wandte sich an letztere: «Sie müssen Vertrauen aufbauen zu den Patienten. Das geht nicht mit Papier.» Es brauche den Austausch unter Angehörigen und die Kommunikation zwischen allen Betroffenen und den Fachpersonen. «Zu glauben, mit einer technischen Verfügung könne man das Lebensende regeln, ist ein Irrglaube», sagte Widler. Er ist der Meinung, der aktuelle "Hype» um die Patientenverfügung, wie er es nennt, sei entstanden aus einem Misstrauen gegenüber den medizinischen Fachpersonen. Aber auch den Fachleuten komme dieses Instrument nicht unbedingt ungelegen. Denn: «Wir machen nicht gerne nichts.» Manchmal aber müsse man etwas eben auch einfach geschehen lassen. Und das brauche Mut. «Als Fachpersonen müssen wir uns mit dem eige- nen Ableben befassen, wenn wir Menschen am Lebensende gut betreuen können wollen. Und wir müssen den Patienten unser Vertrauen geben können, so wie sie uns vertrauen können müssen. Das ist das Wichtigste."

\section{Eine Patientenverfügung, von der niemand weiss, nützt nichts}

Im ersten Referat der Fachtagung zeigte sich Barbara Federspiel, Chefärztin Innere Medizin am Seespital Horgen, mit Widler nicht in allen Aussagen einig. Sie beschäftigt sich seit Jahren intensiv mit Patientenverfügungen und glaubt nicht, dass sie ein Ausdruck des Misstrauens gegenüber dem medizinischen Fachpersonal sind. Ihrer Meinung nach seien sie ein geeignetes Instrument, Wünsche zu formulieren. Im allgemeininternistischen Setting eines Akutspitals, so Federspiel, teilten beim Eintritt ins Spital die wenigsten Patientinnen mit, ob sie eine Verfügung hätten oder

Es braucht klare und realistische Handlungsanweisungen an die Fachleute, wenn es darauf ankommt.

nicht, man müsse unbedingt danach fragen. Dabei findet es Federspiel weniger entscheidend, wie viele Patientinnen tatsächlich eine Patientenverfügung haben - auch wenn es mehr sind als noch vor rund zehn Jahren. Denn: «Keine Patientenverfügung zu haben, kann auch eine Aussage sein", glaubt sie. Entscheidend sei, Verfügungen zu berücksichtigen, wenn sie vorliegen. Für die einfachere Umsetzung von Wünschen aus der Verfügung empfiehlt Federspiel, konkrete Beispiele zu formulieren und zu erklären, was man unter Begriffen wie beispielsweise «dement», «senil» oder "selbständig» genau verstehe. Wichtig sei, dass die Fachpersonen bedenken: "Wir sind vielleicht Experten in unseren Fachgebieten. Aber wir dürfen nicht vergessen, dass die Patienten die eigentlichen Experten für ihr eigenes Wohlbefinden sind.» 


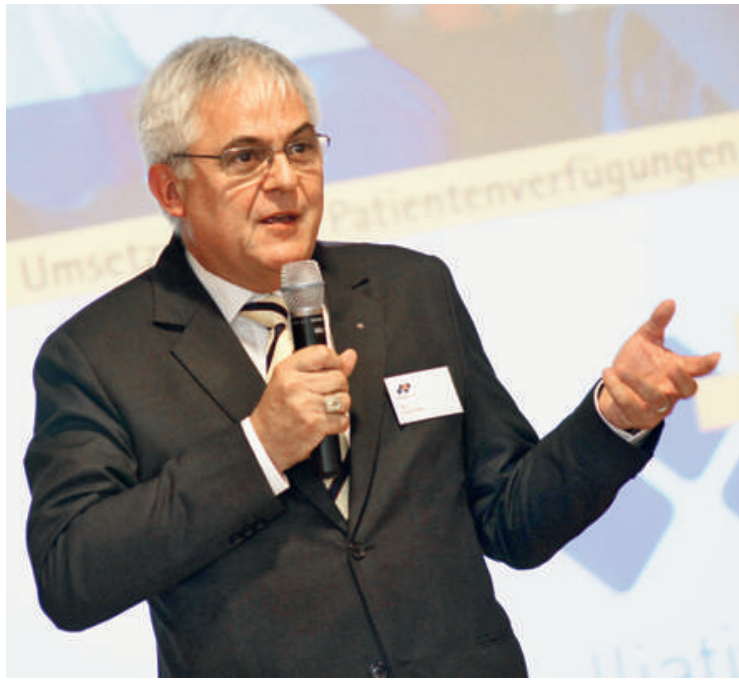

Josef Widler, Präsident der Ärztegesellschaft des Kantons Zürich: "Sie müssen Vertrauen aufbauen zu den Patienten. Das geht nicht mit Papier.»

\section{«Das Thema wird immer weiter hinaus- geschoben"}

Mit diesen ersten Ausführungen war bereits für genügend Diskussionsstoff gesorgt. Am anschliessenden Podiumsgespräch nahmen neben Josef Widler und Barbara Federspiel auch der Palliativmediziner Roland Kunz, die ärztliche Leiterin der Notfallstation am USZ Dagmar Keller Lang, die Psychologin und Beraterin Dorothee Bürgi sowie Patrizia Kalbermatten von «Dialog Ethik» teil. Auf die Frage des Moderators Aschi Rutz, ob die Fachpersonen in der Praxis überhaupt Zeit hätten, die Betroffenen nach einer Patientenverfügung zu fragen, herrschte grosse Einigkeit: Der Zeitmangel sei kein Argument gegen Gespräche, die Fachpersonen mit Betroffenen über das Lebensende führen sollten. Während Widler glaubt, es liege schlicht am Thema und die Fachpersonen gingen diesem einfach lieber aus dem Weg, meinte Keller Lang: «Das Thema wird

Zeitmangel ist kein Argument gegen Gespräche, die Fachpersonen mit Betroffenen über das Lebensende führen sollen.

mit unserer hochspezialisierten und -technisierten Medizin einfach immer wieder hinausgeschoben und verzögert. Am Ende hat man den Zeitpunkt, um übers Sterben zu reden, schlicht verpasst.» Bürgi glaubt, es mangele «eben an dem, was Josef Widler am Anfang angesprochen hat: an Beziehungen und Gesprächen, an der nötigen Haltung». Um Gespräche übers Lebensende für alle zu erleichtern, findet Kunz es wichtig, dass in der Öffentlichkeit über das Lebensende und auch über die Grenzen der Medizin viel mehr gespro- chen wird. Die Teilnehmenden dieser Gesprächsrunde erwähnten auch, dass auf der anderen Seite klar zu akzeptieren sei, wenn Betroffene nicht übers Sterben sprechen oder keine Patientenverfügung ausfüllen wollten. Und jene, die das gerne möchten, wünschten sich sehr oft Unterstützung dabei, ergänzte eine Teilnehmerin aus dem Publikum. «Die Patientenverfügungen müssten einfacher, klarer und lesbarer werden für die Patienten», so Keller Lang.

\section{«Wir sind ja so klug, wenn wir gesund sind»}

Die Juristin Brigitte Tag konnte diesen Faden aufnehmen. Sie betonte, dass es sehr wichtig sei, eine Patientenverfügung regelmässig zu erneuern, damit ihr Inhalt wirklich den aktuellen Wünschen entspreche und sie auch tatsächlich im entscheidenden Moment rechtsgültig sei. Die Variante, sich regelmässig mit Entscheidungsbevollmächtigten über die eigenen Wünsche am Lebensende zu unterhalten, sei mindestens ebenso gut, betonte Tag und sagte: «Das neue Erwachsenenschutzrecht zwingt niemanden zu etwas.» In Situationen, in denen es um Entscheidungen am Lebensende gehe, sei es die Aufgabe des Arztes oder der Ärztin, die Entscheidungsvoraussetzung zu schaffen für die Patientinnen. Dies sei eine sehr wichtige Aufgabe und bedeute eine grosse Verantwortung. Das neue Erwachsenenschutzrecht (seit 2013 in Kraft) wolle mit zwei Instrumenten die Autonomie der Menschen stärken: der Vorsorgevollmacht und der Patientenverfügung. Eine Patientenverfügung ist kein Vertrag, sie kann jederzeit geändert oder vernichtet werden. «Wir sind ja so klug, wenn wir gesund sind. Aber wenn wir krank werden, ändert sich sehr vieles», sagte Tag.

\section{Wie mit kranken Kindern und Eltern übers Lebensende reden?}

Im Anschluss berichtete die Psychologin Rosanna Abbruzzese von ihren Erfahrungen, die sie mit Kindern am Lebensende am Kinderspital Zürich macht. Wer mit Kindern übers Sterben reden wolle, sollte zunächst einmal altersadäquat informieren, so $\mathrm{Ab}$ bruzzese. Sie ging als Beispiel auf das Todeskonzept von Kindern im Vorschulalter ein, die noch davon ausgehen, dass der Tod reversibel sei. Wichtig sei auch, dass Fachpersonen familiäre und kulturelle Einstellungen respektierten. «Respektvoll nach Gründen für bestimmte Verhaltensweisen zu fragen, hilft uns, besser zu verstehen", so Abbruzzese. Sie erwähnte in ihrem Referat auch verschiedene Herangehensweisen und «Offenheitsgrade» in der Kommunikation. Wo es 
möglich sei, direkt verbal übers Sterben zu sprechen und das Kind auch den Willen dazu signalisiere, sei das angebracht. Sei dies nicht der Fall, sei es manchmal möglich, indirekt oder auch symbolisch-bildlich zu kommunizieren. Aber auch die Vermeidung und Verdrängung sei eine Strategie und auch das sei zu akzeptieren.

\section{«Vertrauenskommunikation beginnt nie bei Null»}

Eine sehr wichtige Grundlage für eine gute Kommunikation war im Verlaufe des Morgens dieser Tagung immer wieder zur Sprache gekommen: Vertrauen. Simon Peng-Keller, Theologe und wissenschaftlicher Koordinator der SNF-Projekte «Vertrauen verstehen» und «Hermeneutik des Vertrauens am Lebensende» an der Universität Zürich, berichtete von Erkenntnissen der Vertrauensforschung: In der Palliative Care haben laut Peng-Keller vor allem das Vertrauen in Personen, also in konkrete (professionelle) Helferinnen, und das Vertrauen in Institutionen, also ins medizinische und

\section{Eine Patientenverfügung ist kein Vertrag, sie kann jederzeit geändert oder vernichtet werden.}

rechtliche System, eine grosse Bedeutung. Vertrauen werde prozessual und wechselseitig aufgebaut. Wichtig dabei sei es, fachliche Kompetenz und Bevollmächtigung zu erweisen sowie Wohlwollen und Verständnis zu signalisieren. Im klinischen Kontext, so Peng-Keller, bemesse sich Vertrauenswürdigkeit in Kompetenz, Integrität und Wohlwollen: «Vertrauenskommunikation beginnt nie bei Null.» Fachpersonen bräuchten einen gewissen Vertrauensvorschuss, auf dem sie aufbauen könnten - und den hätten sie auch. Peng-Keller ging auch auf die positiven Folgen einer vertrauensvollen Beziehung ein. Sie entfalte eine heilende Wirkung und bilde eine sehr wichtige Grundlage für die Bewältigung von Angst.

In den zahlreichen anschliessenden Workshops diskutierten die Teilnehmenden über Themen wie Gesprächsführungskompetenz, Gespräche mit demenzbetroffenen Menschen, mit Kindern oder auch mit Menschen mit Migrationshintergrund, über psychologische Aspekte beim Verfassen einer Patientenverfügung, über Advance Care Planning oder über die Symbolsprache Sterbender.

\section{Patientenverfügungen und ihre Grenzen}

Die Workshopleitenden berichteten am Ende von den Themen, die in ihren Gruppen für besonders angeregte Diskussionen gesorgt hatten. So erzählte beispielsweise Andreas Weber, dass man im Workshop "Advance Care Planning" feststellte, dass die derzeitigen Patientenverfügungen in der Praxis oft an Grenzen stossen, weil einiges darin nicht geklärt wird. Eine Notfallplanung, die Betroffene und Fachpersonen gemeinsam erarbeiten, könnte einen grossen Dienst erweisen. Sie könnte bei Patienten und Patientinnen vorgenommen werden, die bereits erkrankt sind und bei denen man aufgrund des Krankheitsbildes als Fachperson abschätzen kann, welche möglichen Notfallsituationen eintreten könnten. Weber setzt mit einem Team von Fachpersonen ein Projekt in diesem Bereich um.

\section{Patientenverfügungen zugänglicher machen: ein konkretes Projekt}

In diesen Zusammenhang passt auch das Projekt, das anschliessend von Patrizia Kalbermatten, wissenschaftliche Mitarbeiterin bei "Dialog Ethik», vorgestellt wurde: ein Projekt für Beratungen zu Patientenverfügungen. «Dialog Ethik», palliative $\mathrm{zh}+$ sh sowie die Ärztegesellschaft des Kantons Zürich planen ein gemeinsames Projekt mit dem Ziel, die Patientenverfügungen zugänglicher zu machen. Damit möchte man einerseits Menschen bestärken, eine Patientenverfügung auszufüllen, wenn sie bereit sind, sich mit dem Thema zu befassen. Andererseits soll vermieden werden, dass widersprüchliche oder unpräzise Aussagen in den Verfügungen festgehalten werden. Man will darum Unterstützung anbieten beim Erstellen einer Patientenverfügung. Dazu sollen einerseits Hausärzte und -ärztinnen sowie Spitex-Mitarbeitende online befragt werden. Andererseits sollen Interviews mit potentiell Verfügenden durchgeführt werden. Die Daten sollen anschliessend eine Grundlage bieten für einen Leitfaden mit Orientierungshilfe zum Verfassen einer Patientenverfügung. Dieser Leitfaden soll in Hausarztpraxen und Spitex-Organisationen verfügbar sein. Zudem ist ein Sensibilisierungsvideo zum Thema geplant, das auf den erhobenen Daten abstützt.

Bildnachweis

palliative $\mathrm{zh}+\mathrm{sh} /$ James D. Walder 